\title{
El objeto yuxtapuesto de las ciencias sociales y sus implicaciones en la formación de docentes
}

\author{
The Juxtaposed Object of the Social Sciences and Its Implications in Teacher Training
}

\section{O objeto justaposto das ciências sociais e suas implicações para a formação de profesores}

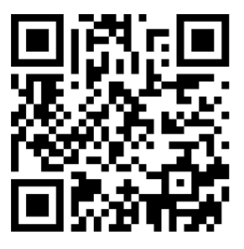

\section{Resumen:}

Objetivo. Analizar el debate sobre la construcción del objeto de las ciencias sociales (en adelante CS) y sus implicaciones en la formación de profesores. Discusión. En un primer momento se propone una conceptualización de las CS a partir de la defensa de la ontología yuxtapuesta de su objeto; asimismo se debaten las consideraciones epistemológicas y metodológicas en clave de totalidad. Posteriormente se reflexiona sobre el lugar del sujeto que practica las CS y sus consecuencias en la producción de conocimiento. Finalmente, se plantean consideraciones en la formación de docentes en CS. Conclusiones. El diseño curricular para formar docentes en CS debe pasar por la construcción de espacios que lleven a problematizar aspectos de la realidad sin reducirlo a compartimentaciones propias de los departamentos. Debe también generar una conciencia epistemológica que les conduzca a dar cuenta de su orden de intelección o política de interpretación en la producción de conocimiento.

Palabras claves: Epistemología de las ciencias sociales; investigación; formación de profesores; enseñanza de las ciencias sociales. 
https://doi.org/10.15359/ree.26-1.25

https://www.revistas.una.ac.cr/index.php/educare

educare@una.ac.cr

\begin{abstract}
:
Objective. To analyze the debate on the construction of the object of the social sciences and its implications in the training of teachers. Discussion. At first, a conceptualization of the social sciences is proposed by defending the juxtaposed ontology of its object; likewise, epistemological and methodological considerations are debated in terms of totality. Then, it is reflected on the place of the subject who practices the social sciences and its consequences in the production of knowledge. Finally, considerations are raised on the training of teachers in social sciences. Conclusions. The curricular design to train teachers in social sciences must go through the construction of spaces that lead to two actions: first, to problematize aspects of reality without being reduced to compartmentalization of the departments; and second, to generate an epistemological awareness that leads them to account for their order of intellection or policies of interpretation in the production of knowledge.
\end{abstract}

Keywords: Social science epistemology; research; teacher training; social science education.

\begin{abstract}
Resumo:
Objetivo. Analisar o debate sobre a construção do objeto das ciências sociais e suas implicações na formação de professores. Discussão. Num primeiro momento, propõe-se uma conceituação das ciências sociais em defesa da ontologia justaposta de seu objeto e as considerações epistemológicas e metodológicas são debatidas na chave da totalidade. Em seguida, reflete-se sobre o lugar do sujeito que pratica as ciências sociais e seus desdobramentos na produção do conhecimento. Por fim, são feitas considerações sobre a formação de professores em ciências sociais. Conclusões. $O$ desenho curricular de formação de professores em ciências sociais deve passar pela construção de espaços que levem a problematizar aspectos da realidade sem se reduzir à compartimentação dos departamentos e gerar uma consciência epistemológica que os leve a dar conta de sua ordem de intelecção ou política de interpretação na produção de conhecimento.
\end{abstract}

Palavras-chaves: Epistemologia das ciências sociais; pesquisa; formação de profesores; ensino das ciências sociais.

\title{
Introducción
}

La formación de docentes en CS enfrenta varios retos metodológicos y teleológicos; especialmente porque se espera que sean los futuros maestros y maestras que eduquen a niños y niñas en saberes, actitudes y habilidades para la democracia, la ciudadanía global y los retos que afronta la humanidad contemporánea (derechos humanos, igualdad, migración, cambio climático, entre otros).

Aunado a esa expectativa, los planes de estudio para la formación de docentes en CS muchas veces no evidencian una clara articulación entre el saber pedagógico, el saber sobre las ciencias, su objeto y la investigación (Rodríguez Ávila, 2013). Esta circunstancias trae como consecuencia una cualificación fragmentada que incide en la persona egresada: o domina conceptos disciplinares pero con una capacidad reducida para la investigación, o bien tiene experiencia en la investigación social pero no tiene un dominio pedagógico que le permita problematizar su práctica y construir saber didáctico que sostenga su enseñanza.

2

Óscar Julián Cuesta-Moreno y Sandra Mireya Meléndez-Labrador

Los artículos de la Revista Electrónica Educare del Centro de Investigación y Docencia en Educación de la Universidad Nacional, Costa Rica, se comparten bajo términos de la Licencia Creative Commons: Reconocimiento, № Comercial, Sin Obra Derivada 3.0 Costa Rica. Las autorizaciones adicionales a las aquí delimitadas se pueden obtener en el correo: educare@una.cr 
Algunos perfiles de egreso de los programas de formación plantean que serán profesionales tanto para la enseñanza y el trabajo en proyectos sociales como para la investigación. Es decir, serán docentes pero también profesionales disciplinares de las CS. Esta proyección no es buena ni mala en sí misma, de lo que se trata es de brindar planes de formación que permitan a maestros y maestras en formación alcanzar esos propósitos.

El presente artículo sostiene que más allá de adecuar los currículos a una agenda de competencias y habilidades profesionales, lo que se debe considerar en primer lugar es la formación epistemológica del maestro y de la maestra en CS (Pagès Blanch, 2011). No se trata de que sean profesionales expertos simultáneamente en antropología, historia, sociología, comunicación, geografía o economía, sino que sean capaces de reflexionar sobre la naturaleza ontológica que articula las CS y desarrollen la capacidad de investigar (participar en la producción de conocimiento disciplinar) y de decidir cuál conocimiento enseñar, para qué enseñarlo y cómo enseñarlo en su práctica profesoral.

Tal formación en la epistemología de las CS también ayuda a superar la distancia que persiste entre la producción de conocimiento científico y lo que se enseña (Aguilera Morales, 2017). Por consiguiente, el maestro y la maestra tendrán elementos para construir rutas de enseñanza que acerquen al estudiantado a saberes, pero sobre todo a los límites y posibilidades del conocimiento social que les alimente un deseo de seguir estudiando (Simons y Masschelein, 2014).

Ahorabien, siloimportanteen la formación de docentes enCS es la reflexión epistemológica, ¿no se afronta un obstáculo aún mayor si el debate sobre su ontología y epistemología no ha logrado un consenso significativo? Precisamente, la primera parte del artículo propone una conceptualización de las CS, para lo cual defiende la ontología yuxtapuesta del objeto de investigación de las CS y señala sus implicaciones epistemológicas y metodológicas en clave de totalidad. En un segundo momento, se muestra el lugar del sujeto que practica las CS y sus implicaciones en la producción de conocimiento. Desde allí se presenta el reto permanente de la configuración de las CS y la dinámica de investigar en ellas. Finalmente, se plantean las consideraciones en la formación de profesores en este campo. Resta decir que el tema es de significativa relevancia en este campo, dado que la formación de maestros y maestras en CS puede estar basada en supuestos que responden a enunciaciones de las modas pedagógicas y no necesariamente en cimientos epistemológicos que tengan más consistencia frente a fluctuaciones que traen las novedades.

\section{La conceptualización de las CS}

Definir las CS es problemático por lo menos por dos óbices. En primer lugar, porque la definición del término ciencias puede quedar anquilosado en la comprensión de ciencia según los preceptos de las disciplinas hipotético-deductivas, de la cual la física se ubica como el mejor ejemplo. En segundo lugar, debido a que bajo la denominación de CS se puede abstraer 
https://doi.org/10.15359/ree.26-1.25

https://www.revistas.una.ac.cr/index.php/educare

educare@una.ac.cr

un conjunto de disciplinas de fronteras porosas (Garcés, 2021) que, incluso, podría incluir a disciplinas humanas (lo que implica afirmar que la clasificación de las ciencias necesita un alto grado de arbitrariedad). En todo caso, sin el ánimo de agotar el debate, a continuación se plantea una definición de ciencias sociales que permita colocar epistemológicamente la formación de docentes en este campo. Para comenzar, se formulará un concepto de ciencias y, basado en esto, una conceptualización de ciencia social.

Sin importar cuál sea su objeto o método, todas las ciencias tienen un elemento común: tienen la intención de hacer inteligible la realidad que, por principio, se resiste a ser alcanzada. Dicho propósito implica un esfuerzo dado que establece un campo disciplinar. El esfuerzo por hacer inteligible la realidad se materializa en conocimiento, es decir, en una representación que abstrae la comprensión y explicación del mundo y brinda horizontes de sentido para vivir en él. En consecuencia, la ciencia puede ser definida como un esfuerzo por producir conocimiento (Sokal, 2009).

El esfuerzo para producir conocimiento, entendido como la representación del mundo basada en la evidencia, la razón y la argumentación (Sokal, 2009), ha decantado algunos elementos para distinguir una ciencia, de los que se pueden destacar: lo ontológico, lo epistemológico, lo metodológico y lo retórico. Lo ontológico hace referencia a la realidad que es estudiada, que podría precisarse en el objeto de investigación, en otras palabras, para ser ciencia se debe tener dicho objeto. Pero este objeto no se encuentra en la realidad inmediata, sino que la ciencia construye, valga decir, conquista, este objeto, por lo que el conocimiento científico implica una ruptura de la satisfacción inmediata que presenta la realidad percibida o sensible (Bachelard, 2000).

Frente a lo epistemológico, la ciencia parte del hecho de que la realidad se resiste a ser asida, de allí que es necesario un esfuerzo por construir una relación consciente con ella. La ontología del objeto construido determinará la relación que el sujeto-constructor guarda con aquel. La epistemología es, entonces, la explicitación de las características de la relación que se guarda con el objeto, así como su sentido, sus vacíos e incertidumbres.

Una vez construido el objeto e identificada su naturaleza (lo ontológico) y explicitada la relación que se guarda con él (lo epistemológico), la ciencia explica la forma en que investiga y produce conocimiento sobre su objeto de estudio, dicho de otra manera, establece lo metodológico, determinando caminos para asir la realidad. No obstante, este proceso no es una secuencia lineal que tiene un final concreto, puesto que la construcción teórica del objeto trae consigo un progreso del método, que al ser aplicado deriva muchas veces en un refinamiento de lo teórico y, al actualizarse esto, de nuevo incide en el desarrollo metodológico (Bourdieu et al., 2002). Esta dinámica no es ajena a la condición humana y por ello se discuten los valores que lo orientan (lo axiológico). 
La ciencia que conquista su objeto y explicita las vicisitudes de la relación que guarda con él, estableciendo modos para investigarlo, también decanta unas formas de expresar y escribir dichos conocimientos; esto se identifica como el elemento retórico. En efecto, la conquista del objeto implica la abstracción de la realidad por conceptos, por lo que investigar en una ciencia implica sumergirse en una forma de enunciación específica. Así, lo retórico son las reglas o principios que ha establecido una ciencia para decir algo sobre el objeto. Consecuentemente, para comunicarse entre científicos hay que seguir las normas que ellos han construido.

No obstante, este proceso de consolidación de una ciencia a nivel ontológico, epistemológico, metodológico y retórico no está ajeno a tensiones, toda vez que "el hecho es conquistado, construido y verificado en y por la comunicación dialéctica entre los sujetos" (Bourdieu, 2003, p. 129), es decir, hay un proceso de construcción colectiva de la verdad a partir de una verificación, transacción, negación y consenso entre pares.

Esta dinámica de puja y consenso es constitutiva de la ciencia a tal punto que, siguiendo a Bachelard (2000, p. 297), "en la obra de la ciencia sólo puede amarse aquello que se destruye, sólo puede continuarse el pasado negándolo, sólo puede venerarse al maestro contradiciéndolo". En ese orden, la ciencia avanza por la posibilidad de poner en duda lo construido, de no quedarse en la complacencia de la explicación alcanzada y de permitirse decir algo más allá de lo establecido. Así, lo ontológico, epistemológico y metodológico son proclives a ser actualizados. Y lo que se mantiene es lo que aguanta el arbitraje: "El conocimiento científico es lo que ha sobrevivido a las objeciones y es capaz de resistir a las objeciones futuras" (Bourdieu, 2003, p. 127).

Lo anterior permitiría afirmar que bajo la denominación de CS se agrupan, entonces, ciencias que han definido un objeto, una epistemología y una metodología a partir de un ejercicio colectivo de consensos resultado de una dinámica proclive a las tensiones. Sin embargo, tal afirmación es difícil de sostener, teniendo en cuenta que, principalmente, la ontología del objeto no es tan impoluta.

En efecto, el objeto de la sociología, el de la antropología, el de la historia, el de la comunicación, el de la educación, el de la economía, por citar algunas de las disciplinas que se aglutinan bajo la categorización de CS, no tiene una definición taxativa que permita establecer límites epistemológicos y metodológicos concretos, ya que el objeto que estudia remite inevitablemente a otros recortes de realidad.

Por ejemplo, si el objeto de estudio de la antropología cultural es la cultura, esta no puede desligarse de los procesos de actualización ya que la cultura no es un sustantivo que se petrifica. De aquí deriva la necesidad de estudiar sus dinámicas temporales (historia). Igualmente, la cultura es resultado de procesos de tensión y hegemonía, por lo que no puede omitirse la sociología. Además, esta cultura se extiende socialmente por procesos educativos y está simbólicamente organizada, por lo que tendría que ser estudiada por la semiótica o la lingüística. 
https://doi.org/10.15359/ree.26-1.25

https://www.revistas.una.ac.cr/index.php/educare

educare@una.ac.cr

La ilustración anterior se podría hacer desde diferentes disciplinas: la producción y acumulación de riqueza no sería un asunto exclusivo de la economía, sino que su comprensión pasaría por la historia, la sociología, la antropología, la geografía, etc. Más aún, es notorio que los debates epistemológicos de una disciplina alimentan los de otra, sin mencionar que a nivel metodológico el asunto de las diferencias es más poroso, tanto así que los manuales no distinguen entre objetos por lo que se comparten las técnicas indistintamente. En efecto, basta revisar cualquier manual de técnicas de investigación en CS para observar que se explican estas técnicas de una manera general, sin especificar diferencias entre, por ejemplo, la realización de una cartografía social para una investigación sociológica, antropológica o la que efectuaría un psicólogo o comunicador. En consecuencia, las supuestas divisiones de las CS"son barreras para un conocimiento mayor en vez de un estímulo para su generación" (Wallerstein, 1999, p. 261), debido a que no tienen lógicas separadas sino, al contrario, tienen un alto entrelazamiento: "ningún modelo de investigación útil puede aislar'factores'conforme a una categoría económica, política y social, y considerar un solo tipo de variable, indicando así de manera implícita que las demás son constantes" (Wallerstein, 1999, p. 262).

¿Quiere decir esto que todas las CS tienen el mismo objeto? ¿O que al final no son ciencias porque no tienen objetos ontológicamente definidos? Sostenemos que las CS son ciencias pero que enfrentan el reto epistemológico de construir objetos que se puedan abstraer en totalidad creciente, articulando el mayor número de recortes y relaciones y no circunscribiéndose únicamente a límites teóricos (Zemelman, 1987).

En efecto, lo que vemos en las CS es una suerte de yuxtaposición, donde las ciencias se colocan juntas sin acatar divisiones (Wallerstein, 1996a). Incluso, el hecho de que el objeto se extienda más allá de compartimentos disciplinares permite pensar una ontología articulada que deriva en un traslape epistemológico y metodológico. Así pues, defendemos que las CS tienen un objeto de investigación de ontología yuxtapuesta, o sea, que se construyen articulando abstracciones categoriales que permiten la relación de conceptos propuestos en diferentes disciplinas. En otras palabras, si el punto de vista crea el objeto (Saussure), el punto de vista de las CS no se encauza por anteojeras infranqueables, sino que, por el contrario, se articula y converge por la ontología compleja de lo que estudia.

Por tanto, frente a la afirmación tautológica de que el objeto de estudio de las CS es la sociedad, no queda más que asentir diciendo que la sociedad está definida por relaciones complejas (Maldonado, 2009), en la medida en que no se pueden simplificar en explicaciones causales, que exigen ser comprendidas desde diferentes perspectivas disciplinares. Estudiar la sociedad remite a un esfuerzo epistemológico de articular perspectivas en clave de totalidad: estudiar al sujeto, las relaciones entre sujetos y de estos con el mundo. Esto implica la construcción de objetos que no se agotan en estantillos teóricos sino que se abren categorialmente a las coyunturas de una realidad en movimiento (Zemelman, 2005). Esta postura está en consonancia con lo señalado por Bachelard (2000) en la medida que el objeto de las ciencias se construye 
haciendo ruptura del sentido común, ya que es resultado de un "sistema de relaciones expresamente construido" (Bourdieu et al., 2002, p. 52).

\section{El sujeto que practica las CS}

Un rasgo singular de las CS es que el investigador hace parte del objeto que construye. Esto implica un reto mayúsculo, pues para construir el objeto hay que hacer una ruptura social (disgregar lo normalizado, naturalizado y simplificado en el sentido común); además, porque al ser las CS una construcción social son resultado de las mismas disputas sociales y, por lo tanto, existe el riesgo permanente de que el investigador imponga su visión de la realidad al punto de tomar "partido en las luchas que describe" (Bourdieu, 2003, p. 153).

Así que la "lucha por la construcción del objeto" (Bourdieu, 2003, p. 154) no puede estar desligada del científico social como sujeto social. Siguiendo a Said (2008), se trata de reconocer que el conocimiento no es neutral y que todavía no se ha inventado un método que permita aislar al investigador de sus "compromisos (conscientes o inconscientes) con una clase, con un conjunto de creencias, con una posición social o con su mera condición de miembro de una sociedad" (p. 31).

Si bien el científico social puede procurar sus investigaciones libres de las restricciones sociales (culturales, históricas, económicas, geográficas, etc.), es necesario aceptar epistemológicamente que "el conocimiento es algo menos parcial que el individuo que lo produce (con sus circunstancias vitales que le enredan y confunden). En consecuencia, este conocimiento no puede ser no político" (Said, 2008, p. 31).

Sin embargo, no se trata de que el investigador tome partida por lo que él considera justo o válido, dado que su subjetividad está, como la de cualquier sujeto, tejida por creencias e ideologías que operan a nivel involuntario. Se trata, entonces, de que el científico social pueda hacer un análisis de sí mismo para, a su vez, analizar los contenidos inconsistentes que"introduce en su práctica" (Bourdieu, 2003, p. 163). De esta manera, cuando el investigador social logra, en ejercicio de retorno sobre sí, hacer cada vez más consciente la dinámica de su construcción subjetiva, social y científica, podrá explicitar el lugar y el interés que orienta su práctica y perspectiva de observación y análisis. Lo objetivo no es acá una supuesta imparcialidad, como si fuera un observador que analiza desde un punto cero (Castro-Gómez, 2010), sino una declaración honesta de intereses.

En ese orden, la ciencia logra de alguna manera colocarle barreras al científico y su condición social (por no decir ideológica o inconsciente) a partir de que el investigador o la investigadora acepte la crítica, el desacuerdo, la verificación, el arbitraje por parte de sus pares. La"objetividad es un producto intersubjetivo del campo científico ... es el resultado del acuerdo intersubjetivo en el campo" (Bourdieu, 2003, p. 146). No obstante, esta labor no es ideal y opera la condición humana: valga poner por ejemplo la trama de intereses y niveles de parcialidad 
https://doi.org/10.15359/ree.26-1.25

https://www.revistas.una.ac.cr/index.php/educare

educare@una.ac.cr

que puede operar en la publicación de artículos, ponencias y libros científicos, así como las estrategias para garantizar el factor de impacto de las publicaciones científicas.

En consecuencia, partimos de reconocer que el científico social es un sujeto que no puede desprenderse de su subjetividad como tejido singular que hila sentidos asidos de su vida social, pero que sí puede hacer un ejercicio de reflexión permanente que le permita hacer consciente (darse y dar cuenta) de los parámetros que orientan su práctica, valga decir, su mirada (Cuesta Moreno, 2018). En esa línea, él debe hacer explícito los intereses que motivan su deseo de saber. Es importante resaltar además que está dispuesto a exponerse en la construcción de conocimiento que haga a sus pares, no simplemente para que ellos validen sus enunciados, sino para que en el encuentro con el otro logre advertir elementos que le permitan seguir ampliando la comprensión de la realidad y de sí mismo como "sujeto-objeto de la investigación como actor social y constructor de conocimiento" (Lander, 2000, p. 12).

Esto implica que el conocimiento que se construye no es para acumular erudición, sino que tiene una dimensión ética y política y puede ser usado para comprender las condiciones sociales e identificar posibilidades para incidir en ellas. En otras palabras, se investiga para determinar lo inédito viable (Freire, 2005) y tener conocimientos que permitan a los sujetos ser protagonistas de su historia, para "reconocer opciones de potenciación y, con base en ello, desarrollar capacidad de reactuación" (Zemelman, 2011, p. 34).

Por tanto, el objeto yuxtapuesto de las CS está indefectiblemente acompañado, así sea con un silencio constitutivo, de estas preguntas: ¿Qué sociedad somos? ¿Por qué somos esta sociedad? ¿Qué sociedad queremos ser? ¿Qué circunstancias son necesarias para lograr dicha sociedad? ¿Qué podemos hacer para que esas circunstancias sean posibles?

Ahora bien, afirmar que el conocimiento producido por el investigador o la investigadora de la CS no solo queda acumulado en artículos y libros, sino que podría coadyuvar en la comprensión de la realidad para incidir en ella, implica hacer explícito un compromiso social con las comunidades y territorios. De allí que hacer CS en Latinoamérica y, por extensión formar docentes en CS, deba pasar por la reflexión permanente por la región, las naciones que la integran y las particularidades al interior de cada país. Por lo que no sobra alentar el mantenimiento y la creación de asociaciones y redes de investigación latinoamericanas en CS y en formación de docentes en este campo.

\section{Las CS y su reto de reconfiguración}

Las CS iniciaron con una intención universalista que hoy no puede tener el mismo nivel de pretensión, toda vez que lo que se diga sobre su objeto de estudio, dadas sus características ontológicas, no necesariamente aplica a todos los grupos sociales. De allí que más bien se hable de un universalismo pluralista que permita interpretaciones articuladas de un mundo complejo e incierto (Wallerstein, 1996b). 
https://doi.org/10.15359/ree.26-1.25

Igualmente, esta pretensión universal no puede desligarse del supuesto metarrelato universal que reduce a las culturas a un proceso desde lo primitivo a lo moderno. Este lente coloca la experiencia histórica europea como único referente, a tal punto que sus construcciones se convierten en "los patrones a partir de los cuales se pueden analizar y detectar las carencias, los atrasos, los frenos e impactos [de las otras sociedades]" (Lander, 2000, p. 10).

Además, dado que la sociedad es resultado de relaciones complejas, las CS tienen el reto de sobrepasar el pensamiento simplificador que tiende a descomponer en partes fáciles de analizar, para evitar la incertidumbre constitutiva de la realidad. Se trata de procurar abordar los problemas desde la complejidad que presentan las interrelaciones entre los seres humanos y de ellos con la naturaleza (Wallerstein, 1996b). Este enfoque implica revisar el lenguaje usado en las CS que puede anquilosar términos tradicionalmente usados pero que no necesariamente dan cuenta de la complejidad de la realidad que se está analizando (atrapados en preceptos ideográficos y nomotéticos) y que no cumplen con abstraer las distintas temporalidades y espacialidades (Wallerstein, 1999).

En efecto, las CS pueden quedar atrapadas por categorías que prefiguran la realidad, como raza, etnia, nación, Estado y familia, sin reconocer que tales conceptos tuvieron un origen circunscrito a un momento teórico, esto es, que tiene constricciones históricas e implican una relación de poder y un sujeto localizado (Said, 2008). No obstante, la reconfiguración de las CS ha traído el uso de categorías originales de otras disciplinas, lo que trae una resemantización que brinda nuevas posibilidades para abstraer y pensar la realidad social. Por ejemplo, Flaherty (2019) sugiere que los sistemas socioecológicos son entidades metabólicas, por lo cual introduce los conceptos de autopoiesis y estructuras disipativas como fundamentales para pensar sobre la organización social y el orden social desde un marco conceptual con potencialmente alto.

Esto implica no solo hacer ejercicio de comprobación y refutación de enunciados (la dinámica de control científico), sino de cuestionar la forma en que se piensa y se organiza la formulación de los enunciados, lo que lleva a cuestionar al propio sujeto cognoscente y su capacidad de pensar la realidad (Zemelman, 1989). De ahí que la persona que investiga puede quedar atrapada en constructos teóricos que cumplen con la retórica y la revisión de pares pero que no brindan elementos para comprender el presente de la realidad. Por ejemplo, en los movimientos sociales y sus actuales dinámicas de organización, el análisis puede estar atado a aparatos conceptuales que no dejan ver lo nuevo y que le exigen rasgos que antaño se esperaban (Flórez Flórez, 2010).

En ese orden, las CS y, por extensión, quien investiga tienen el reto de no enredarse en una red de constructos teóricos que sirvieron en un momento para abstraer la realidad, pero que no necesariamente pueden dar cuenta del movimiento de esta. Es necesario pasar de un pensar teórico a un pensar epistémico que le evite el atrapamiento en enunciados predicativos sino abiertos a posibilidades categoriales conducentes a construir un conocimiento con pertinencia histórica (Zemelman, 2005). El conocimiento científico también se anquilosa y puede convertirse 
https://doi.org/10.15359/ree.26-1.25

https://www.revistas.una.ac.cr/index.php/educare

educare@una.ac.cr

en contenidos naturalizados que prefiguran el mundo y operan como preconceptos (por no decir como estereotipos y prejuicios), por lo que es importante volver al momento de ruptura (Bachelard, 2000; Bourdieu et al., 2002) y a objetivar al sujeto que objetiviza (Bourdieu, 2003).

\section{Investigar en CS}

A partir de lo anterior, se podrían postular algunas consideraciones para la investigación en CS. La primera, la importancia de conquistar el objeto a partir de romper lo reificado usando categorías abiertas al movimiento de la realidad. Por otro lado, que estas categorías permitan la abstracción de relaciones y que puedan, en clave de totalidad, articular varios niveles de la realidad (lo político, económico, cultural, etc.).

Acá quien investiga podría preguntarse, ¿qué concepto permite condensar más relaciones en el mayor número de niveles? Tal concepto tendría un mayor poder de abstracción y tendría más posibilidades de ordenar la mirada del sujeto (y las relaciones que hay entre conceptos). Vista así, la investigación es comprender relaciones, ver que la realidad no es algo dado, sino que se ve obvia, natural, por herencias culturales y biológicas (Maturana y Varela, 2003) y por la necesidad de ver el mundo como regular y predecible (Pozo, 2003). Es decir, investigar es disgregar lo aparentemente sólido y articular lo aparentemente desligado.

Por eso, investigar es investigarse, porque cuando el sujeto efectúa la práctica de investigación termina dándose cuenta que lo que sabía no era tal y que lo que veía estaba atado a condicionantes sociales y culturales, en otras palabras, determinado por regímenes de verdad y hegemonías aceptadas. Por ende, al investigar muchas veces se da la sensación de ver algo que estaba allí pero que antes no sea había visto, porque esta nueva ruptura y categorización lo hace inteligible. En ese orden, hay que considerar la investigación como una práctica que genera la disposición a pensar. Y, de esta manera, que las CS permiten que la sociedad se piense a sí misma y, consecuentemente, tenga elementos para su propia transformación.

\section{Consideraciones en la formación de docentes en CS}

Con base en las consideraciones expuestas, lo primero que hay que decir es que formar docentes implica ofrecerles una ruta curricular ajena a las supuestas compartimentaciones que dividen las CS en departamentos distantes (Wallerstein, 1996b). Por el contrario, se trata de presentarles una formación disciplinar desde el presupuesto de que el objeto de las CS tiene una ontología yuxtapuesta que exige la articulación en clave de totalidad.

Para ello se pueden considerar cursos de formación que fomenten el pensamiento epistémico, procurando no partir de enunciados predicativos y evitando caer en la tendencia de ponerle siempre nombres a los fenómenos. Más bien parece necesario preguntarse por cuántos nombres puede tener (Zemelman, 2005). Esta disposición facilitaría pensar usando y articulando categorías de la antropología, la historia, la sociología, etc. 
Por otro lado, formar docentes en CS implicaría formar sujetos que tienen dos pasiones: el deseo de saber sobre la sociedad y su complejidad y el deseo de enseñarlo a oteas personas. Esto se denomina saber pedagógico, es decir, la capacidad de recontextualizar conocimientos producidos en las CS y "tener la capacidad de reflexionar sobre el qué, con quiénes, cómo, para qué de la educación y el qué, cómo, para qué de la enseñanza" (Cuesta Moreno, 2017, p. 82). Asumir este postulado implicaría de forma explícita ubicar su práctica de enseñanza en una dimensión política.

Para despertar el deseo de saber en el profesorado es importante alimentar una insatisfacción con los discursos establecidos. Dicho de otra manera, alimentar la sospecha de los enunciados que circulan en el campo de las CS y del campo de la pedagogía, para poder lograr esa negación y rupturas que aviven nuevas construcciones. En ese orden, es formar investigadores e investigadoras que puedan inquietarse por la posibilidad de comprender la sociedad como objeto de estudio y el horizonte de su práctica de enseñanza como acción intencional de formar sujetos para la misma sociedad.

\section{Conclusiones}

El presupuesto de que el objeto de las CS tiene una ontología yuxtapuesta parte de un postulado: se requiere construir una relación en clave de totalidad y consecuentemente, una metodología investigativa en clave de articulación. Esto lleva a sostener que la preparación de docentes de CS debe contemplar planes de estudio con espacios de reflexión epistemológica y de experiencias de investigación que vinculen diferentes estamentos disciplinares. Cely Rodríguez y Moreno Lahe (2016), por ejemplo, han propuesto una metodología que articula la enseñanza de la geografía al estudio de la ciudad y la literatura, con la que logran una iniciativa didáctica que dispone a la reflexión a aquellas personas que estudian.

Formar docentes en CS implicaría también la formación de pensadores y pensadoras que tengan por principio que el objeto de investigación no está dado sino que es construido de acuerdo con la perspectiva que cada quien asuma. En otras palabras, implicaría brindarle al estudiantado herramientas epistemológicas y marcos ético-políticos que le lleven a ser explícitos en su perspectiva y no asignar a su punto de vista supuestas asépticas posiciones. Al explicitar el orden de intelección o política de interpretación que tiene logra advertir sus límites y posibilidades en el conocimiento que procura construir.

Finalmente, el diseño curricular para formar docentes en CS debe pasar por la construcción de espacios que lleven a problematizar aspectos de la realidad sin que quede reducido a compartimentaciones propias de las departamentos. También debe contribuir a generar una conciencias epistemológica que les lleve a dar cuenta de su orden de intelección o política de interpretación en la producción de conocimiento. Desde luego, tal diseño curricular debe ser consecuencia de una propuesta pedagógica que explicite sus presupuestos ontológicos, epistemológicos y metodológicos de manera articulada con las concreciones que sustentan las 
https://doi.org/10.15359/ree.26-1.25

https://www.revistas.una.ac.cr/index.php/educare

educare@una.ac.cr

CS. Un proyecto como este derivaría en una enunciación didáctica que aborde el cómo enseñar, y en una reflexión total sobre la enseñanza misma, entendida como práctica que define el estatuto social de los maestros y maestras.

\section{Declaración de Material complementario}

Este artículo tiene disponible, como material complementario:

-La versión preprint del artículo en https://doi.org/10.5281/zenodo.5563080

\section{Referencias}

Aguilera Morales, A. (2017). La enseñanza de la historia y las ciencias sociales hoy: Contrasentidos y posibilidades. Folios, (46), 15-27. https://doi.org/10.17227/01234870.46folios15.27

Bachelard, G. (2000). La formación del espíritu científico. Contribución a un psicoanálisis del conocimiento objetivo. Siglo XXI.

Bourdieu, P. (2003). El oficio de científico: Ciencia de la ciencia y reflexividad. Anagrama.

Bourdieu, P., Chamboredon, J.-C. y Passeron, J.-C. (2002). El oficio del sociólogo. Presupuestos epistemológicos. Siglo XXI.

Castro-Gómez, S. (2010). La hybris del punto cero: Ciencia, raza e ilustración en la Nueva Granada (1750-1816) (2 ed.). Pontificia Universidad Javeriana.

Cely Rodríguez, A. y Moreno Lache, N. (2016). Ciudad y literatura. Una posibilidad para aprender y enseñargeografía. Universidad Pedagógica Nacional.https://doi.org/10.17227/pd.2016.8625

Cuesta Moreno, O. J. (2017). Formación de maestros, campo pedagógico y deseo de saber. Horizontes Pedagógicos, 19(2), 77-84. https://doi.org/10.33881/0123-8264.hop.19203

Cuesta Moreno, Ó. J. (2018). La formación de investigadores sociales desde marcos críticohermenéuticos: Un análisis a la propuesta del Instituto Pensamiento y Cultura en América Latina - IPECAL. Revista Kavilando, 10(2), 356-374. https://www.kavilando.org/revista/ index.php/kavilando/article/view/213

Flaherty, E. (2019) Complexity theory: Societies as complex systems. En Complexity and resilience in the social and ecological sciences (pp. 29-76). Palgrave Macmillan. https://doi. org/10.1057/978-1-137-54978-5_2

Flórez Flórez, J. (2010). Lecturas emergentes: Decolonialidad y subjetividad en las teorías de movimientos sociales. Pontificia Universidad Javeriana.

Freire, P. (2005). Pedagogía de la esperanza. Un reencuentro con la pedagogía del oprimido. Siglo XXI.

12 Óscar Julián Cuesta-Moreno y Sandra Mireya Meléndez-Labrador

Los artículos de la Revista Electrónica Educare del Centro de Investigación y Docencia en Educación de la Universidad Nacional, Costa Rica, se comparten bajo términos de la Licencia Creative Commons: Reconocimiento, No Comercial, Sin Obra Derivada 3.0 Costa Rica. Las autorizaciones adicionales a las aquí delimitadas se pueden obtener en el correo: educare@una.cr 
Garcés, L. J. (2021). ¿Educación, disciplina sin fronteras? Utopía y praxis latinoamericana, 26(94), 128-136. https://dialnet.unirioja.es/servlet/articulo?codigo $=8040409$

Lander, E. (2000). Ciencia sociales: Saberes coloniales y eurocéntricos, perspectivas latinoamericanas. En E. Lander (Ed.), Ciencia sociales: Saberes coloniales y eurocéntricos, perspectivas latinoamericanas (pp. 11-40). Clacso.

Maldonado, C. (2009). Complejidad de los sistemas sociales: Un reto para las ciencias sociales. Cinta de moebio, (36), 146-157. https://doi.org/10.4067/S0717-554X2009000300001

Maturana, H. y Varela, F. (2003). El árbol del conocimiento. Las bases biológicas del entendimiento humano. Lumen Editorial Universitaria.

Pagès Blanch, J. (2011). ¿Qué se necesita saber y saber hacer para enseñar ciencias sociales?: La didáctica de las ciencias sociales y la formación de maestros y maestras. Edetania: Estudios y propuestas socio-educativas, (40), 67-81. https://dialnet.unirioja.es/servlet/ articulo?codigo $=3843517$

Pozo, J. I. (2003). Adquisición de conocimiento. Morata.

Rodríguez Ávila, S. P. (2013). Aproximación a la formación de profesores en ciencias sociales y en historia en Colombia: Elementos para el debate. Reseñas Enseñanza de la Historia, (11) 29-56. https://apehun.uncoma.edu.ar/index.php/revista-resenas

Said, E. (2008). Orientalismo. Random House.

Sokal, A. (2009). Más allá de las imposturas intelectuales. Ciencia, filosofía y cultura. Paidós.

Simons, M. y Masschelein, J. (2014). Defensa de la escuela: Una cuestión pública. Miño y Dávila.

Wallerstein, I. (1996a). Abrir las ciencias sociales. Revista Colombiana de Educación, (32). https:// doi.org/10.17227/01203916.7759

Wallerstein, I. (Coord.). (1996b). Abrir las ciencias sociales. Informe de la Comisión Gulbenkian para la reestructuración de las ciencias sociales. Siglo XXI.

Wallerstein, I. (1999). Impensar las ciencias sociales: Límites de los paradigmas decimonónicos. Siglo XXI.

Zemelman, H. (1987). La totalidad como perspectiva de descubrimiento. Revista Mexicana de Sociología, 49(1), 53-86. https://doi.org/10.2307/3540427

Zemelman, H. (1989). Hacia una reflexión sobre las Ciencias Sociales en América Latina. Estudios Latinoamericanos, 4(6-7), 11-17. https://doi.org/10.22201/cela.24484946e.1989.6-7.47384

Zemelman, H. (2005). Voluntad de conocer. El sujeto y su pensamiento en el paradigma crítico. Antrhopos.

Zemelman, H. (2011). Los horizontes de la razón III. Elorden delmovimiento. Anthropos. Universidad de Manizales 